\title{
Identification of lateral gene in the genomic DNA sequence, by using the $G+C$ content at the third position of synonymous codons of orthologous genes as an index
}

\author{
Yuko Ohfuku $^{1,2,3}$, Hideo Tanaka $^{2}$ and Masami Uebayasi ${ }^{3}$ \\ ${ }^{1} \cdot$ National Institute of Evaluation and Technology, 2-49-10 Nishihara Shibuyaku Tokyo, 151-0066 \\ Japan \\ 2. Agricultural Sciences University of Tsukuba, 1-1-1 Ten-nodai Tsukuba city Ibaraki, 305-8572 Japan \\ 3. Institute of Molecular and Cell Biology, AIST Central 6, 1-1-1 Higashi Tsukuba Ibaraki, 305-8866 \\ Japan \\ E-mail: ohfuku-yuko@meti.go.jp
}

(Received April 25, 2002 ; accepted June 10, 2002 ; published online July 29, 2002)

\begin{abstract}
All open reading frames (ORFs) of 6 Archaebacterial and 17 Eubacterial species showed a strong positive correlation between the $G+C$ content (GC content) of the genomic DNA sequences and the $G+C$ content of the third position of the codons (GC3 content). Among them, 1217 pairs of genes that are orthologous between Pyrococcus horikoshii OT3 (Ph) and Pyrococcus abyssi $(\mathrm{Pa})$ were identified. The codons of each pair of orthologous genes were classified into three categories, identical codons coding for the same amino acid (IC), different codon coding for the different amino acids (DC) and synonymous codons coding for the same amino acids (IA). In a comparison of the GC3 content of these three types in all orthologous genes between $P h$ and $P a$, the GC3 content of IA (GC3 content of synonymous codons) deviated the most from the expected value for the GC content of the genome sequences used in this analysis. Therefore the GC3 content of synonymous codon was suggested to be an index that would be able to distinguish a lateral gene from the orthologous genes of the genomic DNA sequence.

By analysis of the GC3 content of synonymous codons, two remarkable regions were found in the genome sequence of $P h$. In one region between $300 \mathrm{Kbp}$ and $420 \mathrm{Kbp}$, the genes have a higher GC3 content in the synonymous codon, and in the other between $1,320 \mathrm{Kbp}$ and 1,400 Kbp, the genes have a lower GC3 content in the synonymous codons. In the former region, twelve of sixteen orthologous genes were homologous to genes of Eubacteria or Archaebacteria. Especially, four orthologous genes showed homology to genes related to synthesis of the cell wall or lipopolysaccharides from Streptococcus. These four genes would be suggested to be lateral genes and to represent a lateral region from Eubacterial species. In the latter region, eight out of thirteen orthologous genes were homologous to genes of Archaebacteria or Eukarya. Six of these eight orthologous genes show homology to the genes related to the function of translation. These genes would be indicated as the stable genes in the progress of evolution, after the divergence between Pyrococcus horikoshii OT3 and Pyrococcus abyssi.
\end{abstract}


Key Words: $\mathrm{G}+\mathrm{C}$ content, synonymous codon, $\mathrm{G}+\mathrm{C}$ content at the third position of the codon, lateral gene

Area of Interest: Bioinformatics and Bio-Computing

\section{Introduction}

The number of the genomes whose DNA sequences have been completely determined recently is increasing very rapidly and DNA sequences of more than thirty genomes are now available. By comparison of the genomic DNA sequences between Archaebacteria and Eubacteria, it was clear that the phylogenetic relationship among them is more complex than expected [1]. Generally, the evolutionary relationship among organisms is explained on the basis of phylogenetic trees derived from the amino acid sequences, coded by genes such as ribosomal RNA genes or other well-conserved genes. But this explanation occasionally leads to a contradiction since different phylogenetic trees would be generated when the different genes are used. To explain this contradiction, a horizontal or lateral gene transfer model was proposed, which stated that in the process of evolution, gene transfer occurred many times among species [2]. Therefore, to better understand the evolutionary relationships among organisms, it is important for such lateral genes to be distinguished from the phylogenetically stable genes of the organism.

One example of lateral gene transfer is the $\mathrm{kfiD}$ gene, which encodes the $\mathrm{K} 5$-specific UDP-glucose dehydrogenase of Escherichia coli. The $\mathrm{G}+\mathrm{C}$ content ( $\mathrm{GC}$ content) of this gene is much lower than that of the genome's DNA sequence. The pattern of the synonymous codon substitutions between $\mathrm{KfiD}$ and Cap3A was different in spite of them having almost equal evolutionary distances. In contrast, the codon usage between KfiD of E. coli and that from Streptococcus was similar. Based on these results, it was assumed that the $k f \mathrm{D}$ gene was transferred from Streptococcus into E. coli [3]. To identify horizontal gene transfer, however, this method requires a database of homologous genes. And this method can detect only one gene as the horizontally transferred gene. Therefore it is necessary that lateral genes in the genomic DNA sequence are systematically identified by means of an index, which will enable the distinction of a lateral gene from the stable genes.

Bellgard M. I. and Gojobori T. showed that the deviation of the $\mathrm{G}+\mathrm{C}$ content at the third position (GC3 content) of IA (GC3 content of synonymous codons) of orthologous genes between Mycoplasma genetalium and Mycoplasma pneumoniae was the largest among the GC3 contents of IC, DC, and IA [4]. They suggested that this bias of the GC3 content of synonymous codons would represent a direct reflection of the evolutionary pressure working on the genome's DNA sequence.

In this paper, we report that the lateral genes among the orthologous genes in Pyrococcus horikoshii OT3 were identified, by using the bias of the GC3 content of synonymous codons as the index to distinguish a lateral gene from the stable genes. Two Pyrococcus genomic DNA sequences, those of Pyrococcus horikoshii OT3 and Pyrococcus abyssi, were chosen since their size is small and they are closely related species. 


\section{Material and Methods}

The twenty-three genomic DNA sequences of Archaebacteria and Eubacteria used in this analysis were downloaded from the NCBI web site (Table1) (ftp://ncbi.nlm.nih.gov/ genbank/genomes/bacteria/).

Table1. Complete genomic DNA sequences used in this analysis

\begin{tabular}{|c|l|c|c|l|l|}
\hline No. & \multicolumn{1}{|c|}{ Organism } & Domain* & $\begin{array}{c}\text { Size } \\
(\mathrm{Mbp})\end{array}$ & Abbreviation & References \\
\hline \hline 1 & Aeropyrum pernix & A & 1.67 & Aero & {$[5]$} \\
\hline 2 & Archaeoglobus fulgidus & A & 2.18 & Aful & {$[6]$} \\
\hline & $\begin{array}{l}\text { Methanobacterium } \\
\text { thermoautotrophicum }\end{array}$ & A & 1.75 & Mthe & {$[7]$} \\
\hline 4 & Methanococcus jannaschii & A & 1.66 & Mjan & {$[8]$} \\
\hline 5 & Pyrococcus horikoshii OT3 & A & 1.80 & Pyro & {$[9]$} \\
\hline 6 & Pyrococcus abyssi & A & 1.8 & Pabyssi & {$[10]$} \\
\hline 7 & Aquifex aeolicus & B & 1.50 & Aquae & {$[11]$} \\
\hline 8 & Bacillus subtilis & B & 4.20 & Bsub & {$[12]$} \\
\hline 9 & Borrelia burgdorferi & B & 1.44 & Bbur & {$[13]$} \\
\hline 10 & Chlamydia pneumoniae & B & 1.23 & Cpnue & {$[14]$} \\
\hline 11 & Chlamydia trachomatis & B & 1.05 & Ctra & {$[15]$} \\
\hline 12 & Deinococcus radiodurans & B & 3.28 & Dra & {$[16]$} \\
\hline 13 & Escherichia coli K-12 & B & 4.60 & Ecoli & {$[17]$} \\
\hline 14 & Haemophilus influenzae Rd & B & 1.83 & Hinf & {$[18]$} \\
\hline 15 & Helicobacter pylori & B & 1.66 & Hpyl & {$[19]$} \\
\hline 16 & Helicobacter pylori & B & 1.64 & Hpyl99 & {$[20]$} \\
\hline 17 & Mycoplasma genitalium & B & 0.58 & Mgen & {$[21]$} \\
\hline 18 & Mycoplasma pneumoniae & B & 0.81 & Mpneu & {$[22]$} \\
\hline 19 & Rickettsia prowazekii & B & 1.10 & Rpxx & {$[23]$} \\
\hline 20 & Synechocystis sp.PCC 6803 & B & 3.57 & Synecho & {$[24]$} \\
\hline 21 & Thermotoga maritima & B & 1.80 & Tmar & {$[25]$} \\
\hline 22 & Treponema pallidum & B & 1.14 & Tpal & {$[26]$} \\
\hline 23 & $\begin{array}{l}\text { Mycobacterium tuberculosis } \\
\text { H37Rv }\end{array}$ & B & 4.40 & Mtub & {$[27]$} \\
\hline
\end{tabular}

* Characters A and B indicate Archaebacteria and Eubacteria, respectively.

Both the $\mathrm{G}+\mathrm{C}$ content of the genomic DNA sequences and the $\mathrm{G}+\mathrm{C}$ content at each codon position for all ORFs of each species were calculated. All ORFs of Pyrococcus horikoshii OT3 (Ph) and Pyrococcus abyssi $(\mathrm{Pa})$ were translated into amino acid sequences. A homology search was done in the non-redundant database (ftp://ftp.ncbi.nih.gov/blast/db/) with each translated ORF of $P h$, using FASTA program [28]. Furthermore, a homology search was done for all the ORFs of $P a$ with each ORF of $P h$ and vice-versa. Pairs of orthologous genes, including one gene from $P h$ and 
one gene from $\mathrm{Pa}$, were chosen. The pairwise alignment of each pair of orthologous genes was done by using CLUSTAL W [29] and then was applied to calculate the GC3 content in the orthologous genes of the two Pyrococcus species. In addition, all ORFs were classified into three domains (Archaebacteria, Eubacteria and Eukarya), to which the homologous genes belong to and/or into eight categories by function, which the homologous genes have [30].

\section{Results and discussion}

3.1 The correlation between the $G+C$ content of the genomic DNA sequences and the $G+C$ content at the third codon position (GC3 content) for all ORFs of the genomic DNA sequences

As shown in Fig.1, the G+C content of twenty-three genomic DNA sequences show the range to be between approximately 25 and 70 percent.

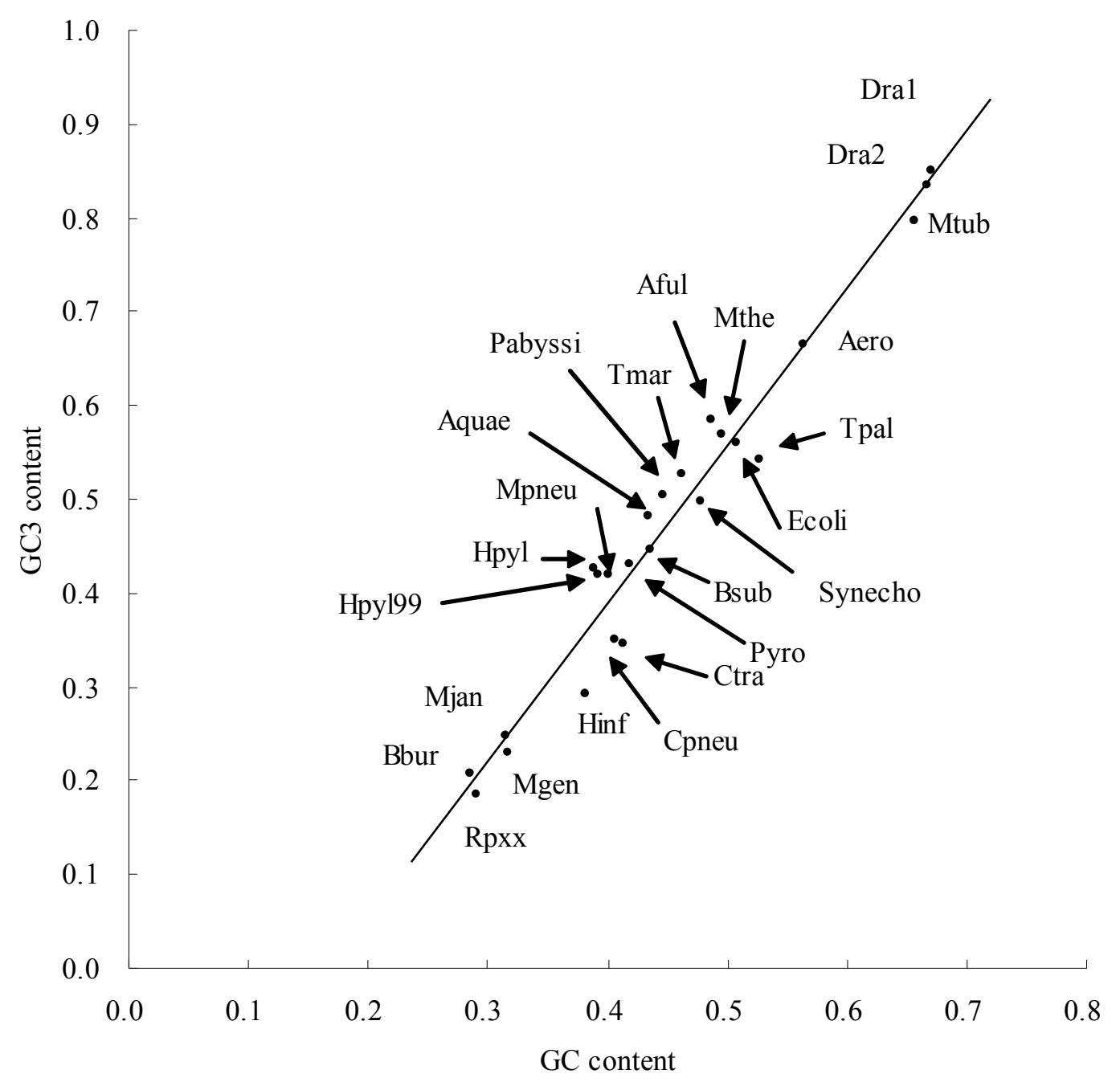

Figure 1. Plot of the $\mathrm{G}+\mathrm{C}$ content between the complete genome sequence (GC content) and the third codon position of all the ORFs (GC3 content).

Abbreviations are as listed in Table 1. A regression line is fitted to the data. 
There was a strong positive correlation, however, reported between the GC content of the genome sequence and the GC content at each codon position in all ORFs of each genome DNA sequence (GC3-plot named in ref [4]) [31]. Nevertheless, the GC3 content in all ORFs demonstrated the best correlation as shown in Fig. 2, which was similar to the result described in ref [32]. Consequently, the use of the GC3 content as a gene index of the gene is suggested to lead to better analytic results, since the genes are thus better patterned and standardized.

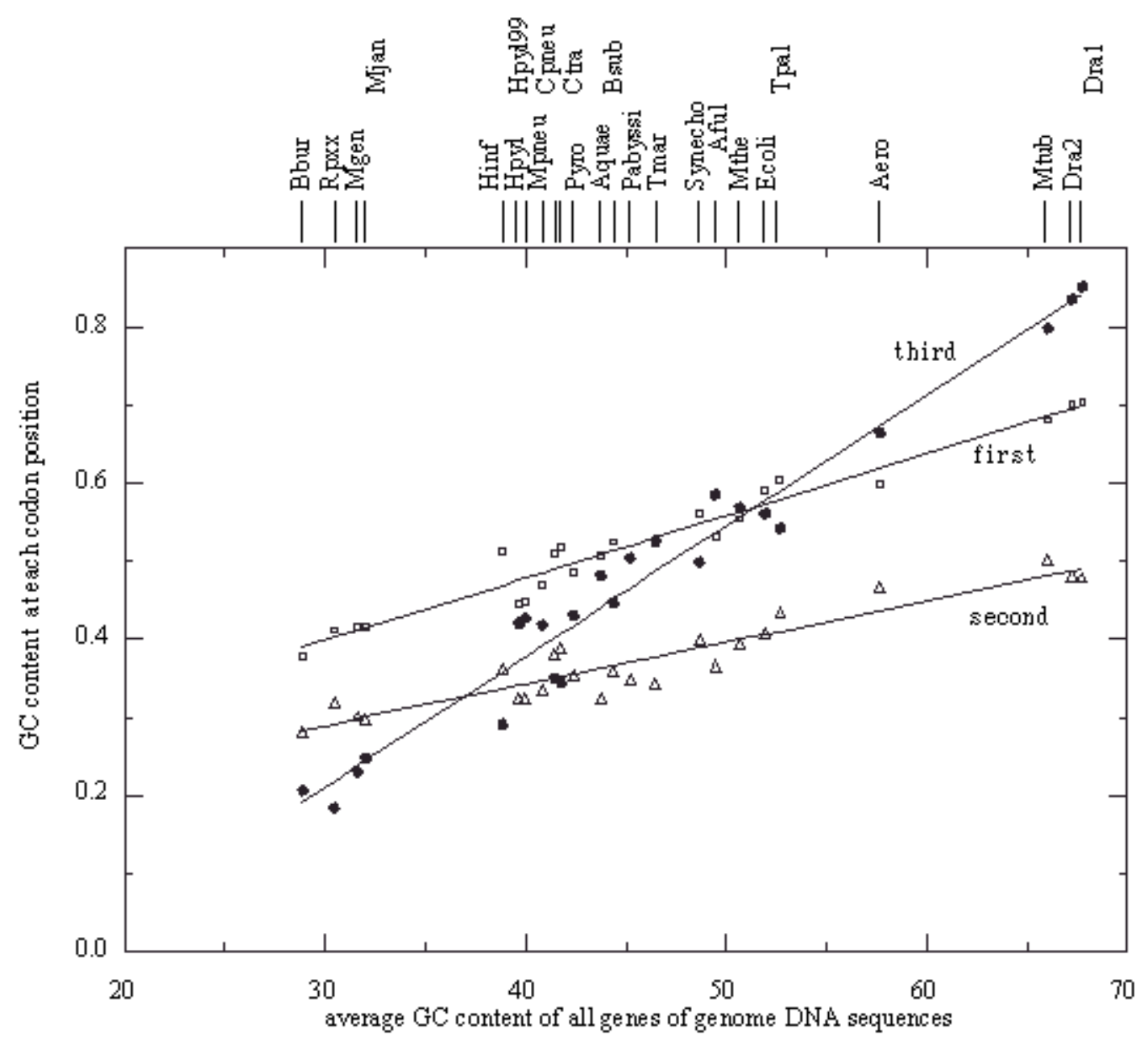

Figure 2. Plots of the average $\mathrm{GC}$ contents at each codon position for all genes against the $\mathrm{G}+\mathrm{C}$ contents of the genomic DNA sequences used in this analysis.

The regression lines were fitted to the data. Open squares $(\square)$, open triangles $(\triangle)$ and filled circles ( $)$ indicate the average $\mathrm{G}+\mathrm{C}$ content of the first, second and third codon positions, respectively. Abbreviations are as listed in Table1. 


\subsection{Definition of the orthologous genes}

Orthologous genes are those having the same function but existing in the different species. Therefore, it is reasonable to suggest that the amino acids sequences coded by orthologous genes would show high homology and nearly similar lengths. Thus, expectation value obtained from the FASTA homology search (E value), which indicates the probability of the error in the FASTA homology search results, and the ratio of the overlap length between them. To compare the pairwise alignment, pairs comprising of one gene from $\mathrm{Ph}$ and one gene from $\mathrm{Pa}$ were chosen. The number of orthologous gene pairs was maximal when the E value was $10^{-20}$, and the ratio of the overlap length between a pair of the orthologous genes was 0.7 . Each pairwise alignment was performed by using the CLUSTAL W program, and the pattern of the homology between them was confirmed. In this way, 1217 pairs of the orthologous genes were identified. The identified orthologous genes were utilized for further analysis.

\subsection{The correlation between the $G+C$ content of the genomic DNA sequences and the $G+C$ content at the third position of the three types of codons found in orthologous genes}

Codons compared between orthologous genes were classified into three categories, IA, DA, and IC. IC indicates the identical codons coding the same amino acids; DC indicates the different codons coding the different amino acids; and IA indicates the synonymous codons coding the same amino acids (synonymous codons).

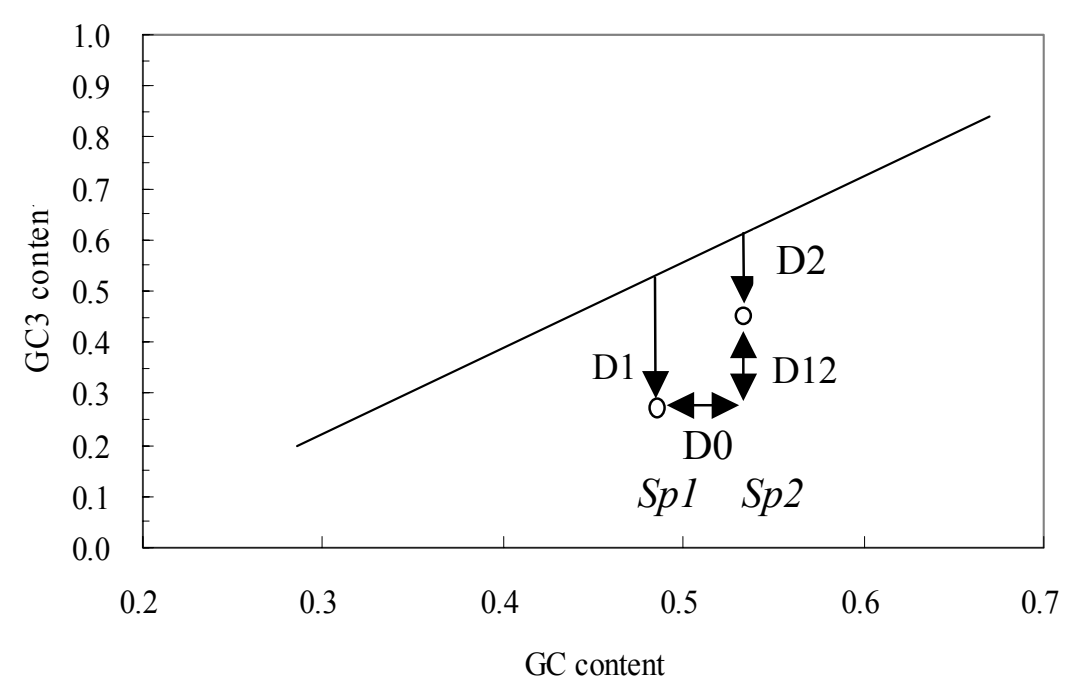

Figure 3. Illustration of the quantities of the differences of GC content used in this analysis. The circles represent the GC3 content for species 1 (Sp1) and species 2 (Sp2), as labeled. D0 represents the difference of the GC content between $S p 1$ and $S p 2$. D1 and D2 indicate the difference of GC content in the overall GC content between $S p 1$ and $S p 2$ from the GC3-plot, respectively. D12 indicates the difference of GC3 content between $S p 1$ and $S p 2$. 
As shown in Fig. 3, D0 indicates the differences of GC content between species 1 and species 2 and D1, D2 indicate the bias of the GC3 content between species 1 and the value calculated using the regression line at the GC content of species 1 and that of species 2 and the value calculated using the regression line at the GC content of species 2. And D12 showed the difference of GC3 content between species 1 and species 2 .
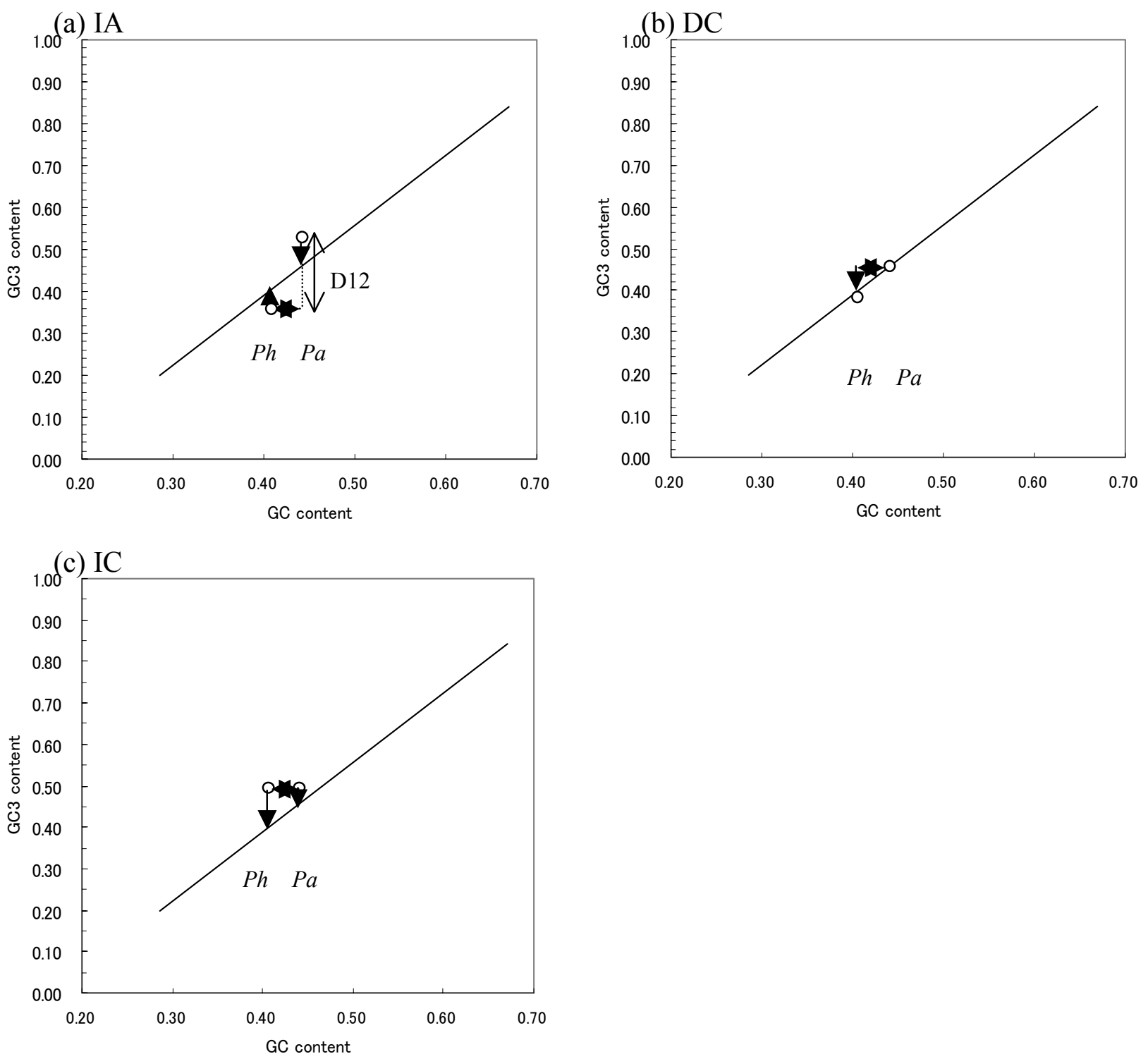

Figure 4. Separate plots of the GC3 content of IA, DC and IC separately, for $P h / P a$ comparison in GC3-plot. $\quad P h$ is species 1 and $P a$ is species 2. (a) IA category. (b) DA category -only D0 and D12 are shown. (c) IC category - D12 is not shown since D12 is zero by definition.

Fig. 4 shows the correlation between the GC3 content of IC, DC, and IA in the orthologous genes among $\mathrm{Ph}$ and $\mathrm{Pa}$ and the GC content of the genomic DNA sequences. The CG3 content of IC is not taken into consideration since the GC3 content does not vary between the two species. The 
deviation of the GC3 content of DC was similar to the expectation value of the GC content of the genomic DNA sequences. The deviation of the GC3 content of IA was the largest. Therefore, the GC3 content of the synonymous codon in the orthologous genes was suggested to distinguish lateral gene from the stable genes of the genomic DNA sequence.

\subsection{Distribution of the $G+C$ content at the third position of synonymous codons in the orthologous genes of $\mathrm{Ph}$}

Figure 5 plots the distribution of the GC3 content of synonymous codons in the orthologous genes against the position of the genomic DNA sequence of $P h$. The average of the GC3 content of synonymous codon in the orthologous genes was 0.365 . The genes having synonymous codons with the higher GC3 content were located particularly in the region from approximately $300 \mathrm{Kbp}$ to $420 \mathrm{Kbp}$ (high GC3 content region). And the genes having synonymous codons with the lower GC3 content were located in the region from approximately $1,320 \mathrm{Kbp}$ to $1,400 \mathrm{Kbp}$ (low GC3 content region).

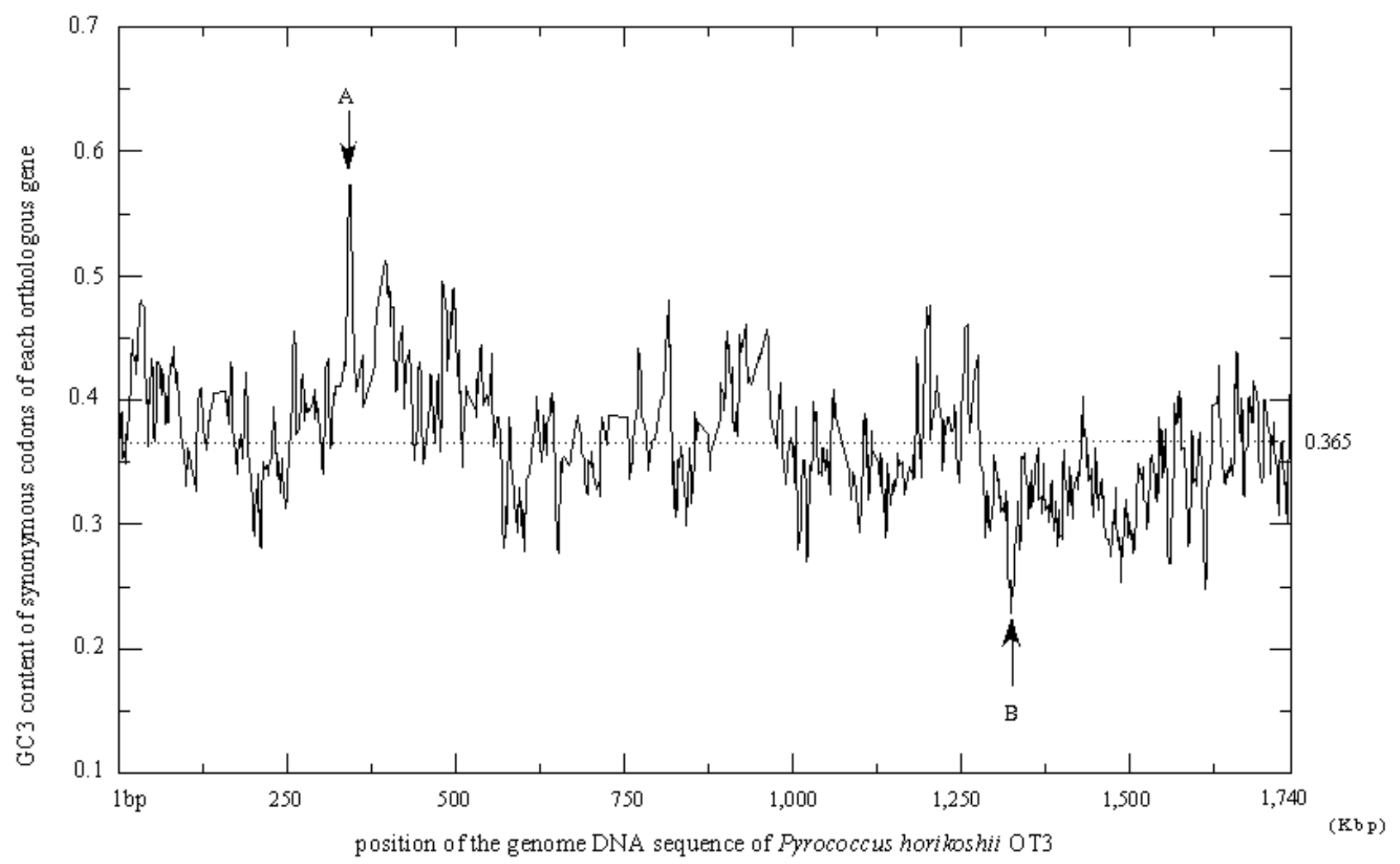

Figure 5. Plot of the GC3 content of synonymous codon of orthologous genes against the genomic DNA sequence of $P h$. A and B indicate the high GC3 and low GC3 content region, respectively. The overall average of the GC3 content of synonymous codons is 0.365 . 
As shown in Fig. 6 (a), the distribution of GC3 content of orthologous genes in $P h$ was regarded as a Gaussian distribution having an average and standard deviation of 0.365 and 0.0709 , respectively. It was reported that 780 genes in the DNA sequence of Escherichia coli, which was corresponded to approximately thirty percent of the whole genome sequence, were analyzed by Fractional Correspondence Analysis and the dynamic clustering method and the genes were classified into three classes [33][34]. The 111 genes constituting the horizontally inherited transfer genes comprised the class with the smallest codon bias among the three classes [34]. Therefore, the score of 0.142 with respect to the total genes analyzed, suggests these genes to be transferred in this DNA sequence. When this value is applied to the distribution of GC3 content of the orthologous genes, the coefficient of the standard deviation is approximately 1.5. Thus, the values of the GC3 content were 0.471 and 0.233 . For the distribution of the GC3 content of putative lateral genes, it might be likely that the curve would increase and decrease near both edges of the distribution. In addition, patterns of the GC3 content of the synonymous codons in both regions as shown in Figure 6 (b), were different from that of all orthologous genes in the genomic DNA sequence. Therefore, by exploring such values that the distribution curve would increase and decrease near both edges of the distribution curve for GC3 content of orthologous genes, we chose the two values, both 0.475 and 0.245 to distinguish the genes of high GC3 content and low GC3 content, respectively, from the orthologous genes. These values were then applied as thresholds for distinguishing lateral genes from the stable genes of $P h$. 
(a) Histogram of GC3 content of synonymous codon of all orthologous genes

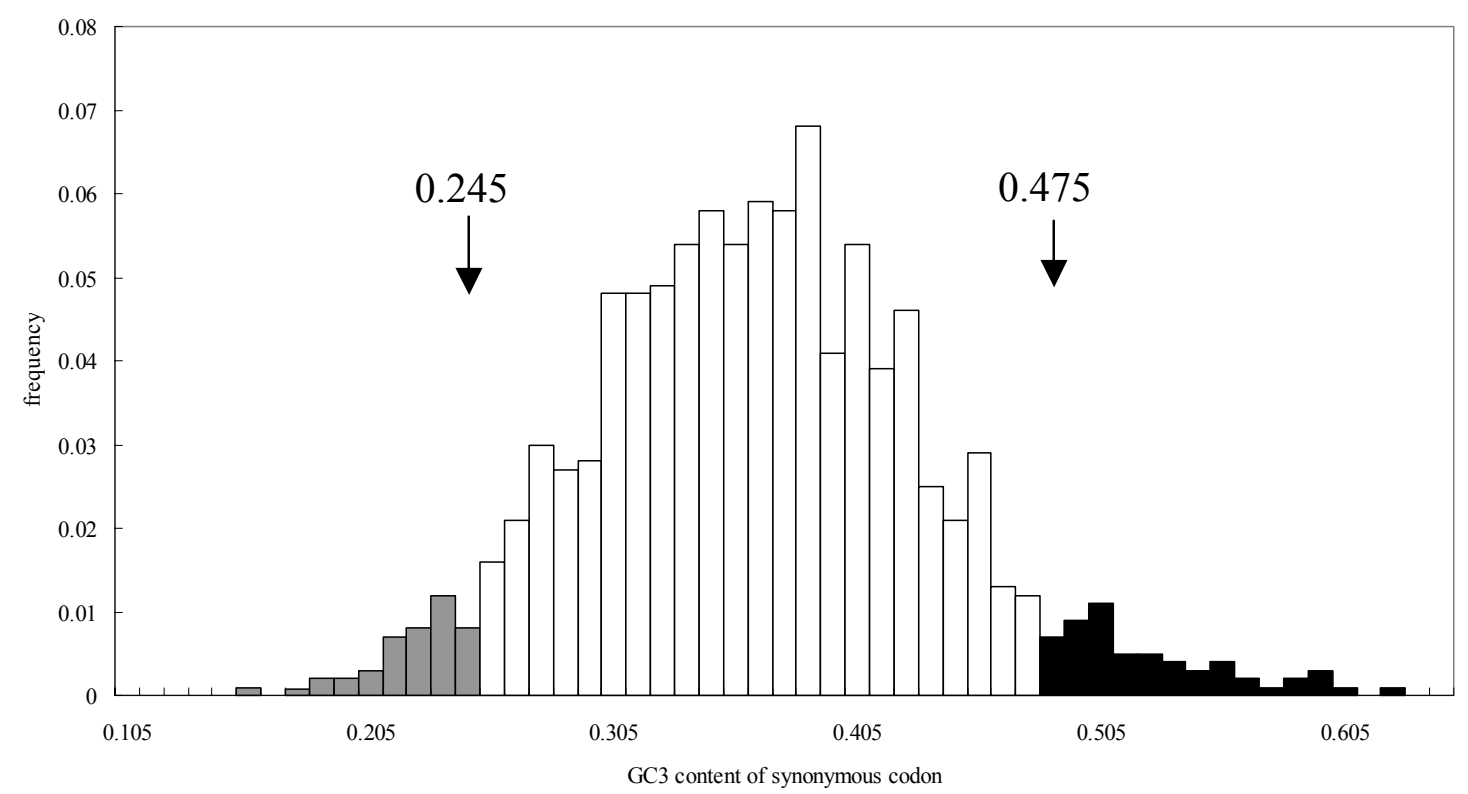

(b) comparison of distribution of $\mathrm{GC} 3$ content of synonymous codon

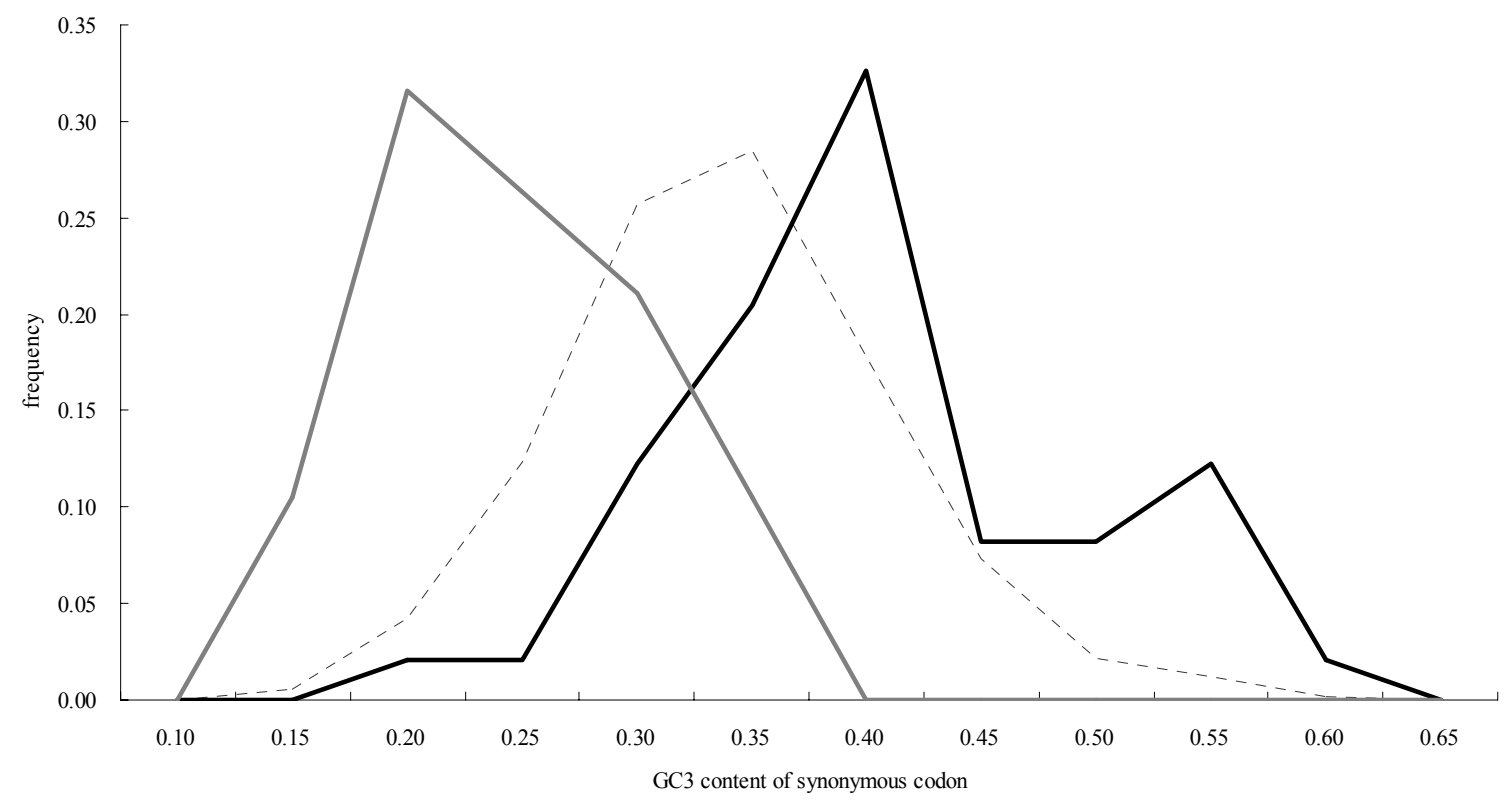

Figure 6. Distribution of the GC3 content of synonymous codons of all orthologous genes in $P h$.

(a) The histogram of GC3 content of synonymous codons of all orthologous genes.

(b) The distribution of GC3 content of synonymous codons of all orthologous genes, the orthologous genes in the region from 300 to $402 \mathrm{Kbp}$, and those in the region from 1,320 to $1,340 \mathrm{Kbp}$, as represented by a gray dashed line, a black bold line and a gray bold line, respectively. 
In the genomic DNA sequence of $P h$, there are 78 orthologous genes, whose GC3 content of the synonymous codons is more than or equal to 0.475 . Sixteen genes were located in the high GC3 content region in Figure 5, region A. In 48 of the orthologous genes, however, the GC3 content of synonymous codons is less than or equal to 0.245 . Thirteen genes were located in the low GC3 content region in Figure 5, region B.

\subsection{Characteristics of the high and low GC3 content regions}

By analysis of one hundred and twenty-six orthologous genes, forty orthologous genes were homologous to genes from Eubacteria and Archaebacteria, twenty-four genes from Eukarya and Archaebacteria and fifty-four from Archaebacteria. Eight orthologous genes had homologous genes from all three domains, Archaebacteria, Eubacteria and Eukarya as shown in Fig. 7.

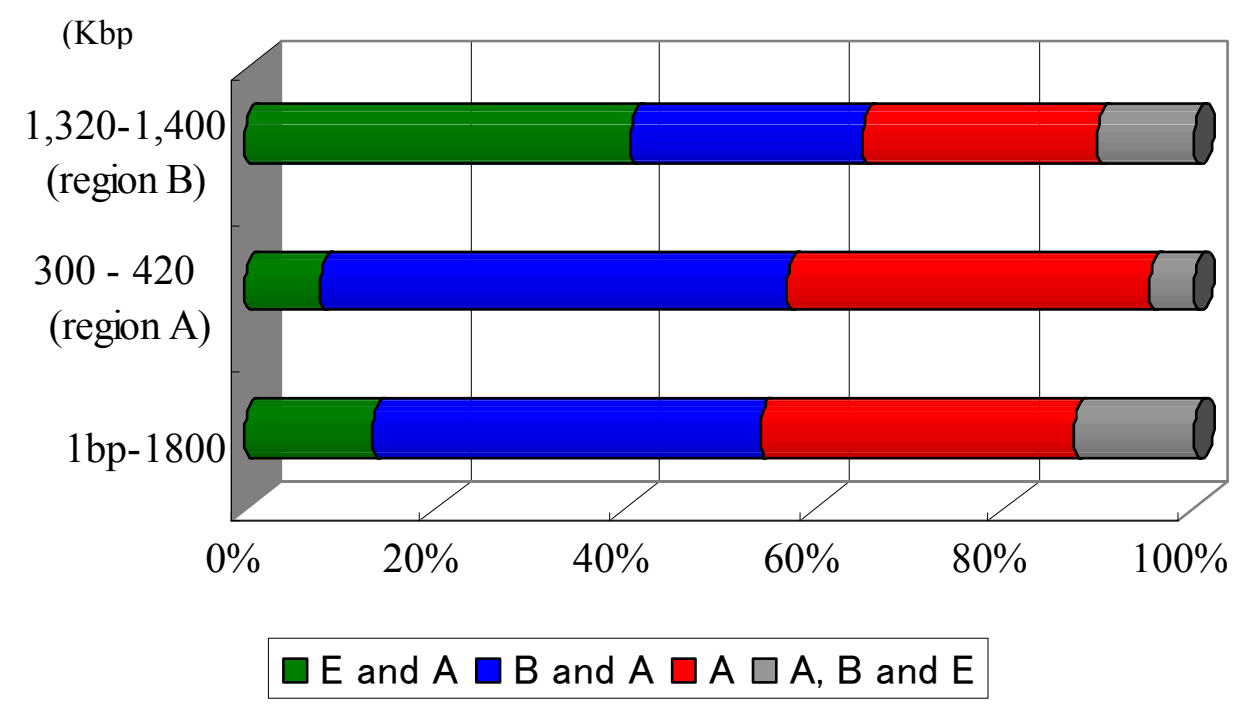

Figure 7. Distribution of the three domains of genes homologous to the orthologous genes in $P h$. Characters E, A and B indicate Eukarya, Archaebacteria and Eubacteria, respectively.

In the high GC3 content region shown in Figure 5, region A, 63 genes were orthologous genes, $49 \%$ and $8 \%$ of which showed homology to the genes from Eubacteria and Archaebacteria and to those of Eukarya and Archaebacteria, respectively. Twelve of 16 orthologous genes, whose GC3 content of synonymous codons is greater than or equal to 0.475 , were homologous to the genes from Eubacteria and Archaebacteria. In the low GC3 content region shown in Figure 5, region B, 69 genes were orthologous genes, $41 \%$ and $25 \%$ of which were homologues to the genes from Eukarya and Archaebacteria and from Eubacteria and Archaebacteria, respectively. Twelve of thirteen orthologous genes, whose GC3 content of synonymous codons is less than or equal to 0.245 , were homologous to the genes from Archaebacteria and Eukarya.

Four orthologous genes in the high GC3 content region, region A as shown in Table 2 (a), were classified into the category of cell structure, because they were homologous to genes related to cell wall or lipopolysaccharides. They also showed high homology to genes related to the cell envelope or biosynthesis of surface polysaccharides and lipopolysaccharides from Streptococcus. 
On the other hand, the organization of the operon related to this function is known to differ among Streptococcus thermophilus species [35]. The $13.6 \mathrm{Kbp}$ epsL-IS981SC region of Streptococcus thermophilus CNRZ386 is closely related to the functionally similar sequence of Lactococcus lactis NIZB40. The IS elements (ISS1 and IS981) shared more than $98 \%$ identity to the homologous ISS1 and IS981 from L. lactis. In addition, probes isolated from this region hybridized with various DNA fragments of L. lactis and the GC content in this region showed variability. This suggests that the entire epsL-IS981SC region was transferred from L. lactis [35]. The four orthologous genes had a bias of GC3 content of synonymous codons and were homologous to the genes from Eubacteria and Archaebacteria in Fig. 8 (b), region A. We found that they are likely to be lateral genes.

Table 2. Number of the GC3 content of synonymous codons of orthologous genes in $\mathrm{Ph}$

(a) GC3 content of synonymous codons of the orthologous genes is 0.475 or greater.

\begin{tabular}{|l|c|c|}
\hline \multicolumn{1}{|c|}{ category of function } & $\begin{array}{c}\text { number of the orthologous } \\
\text { genes }\end{array}$ & $\begin{array}{c}\text { number of the orthologous genes in the } \\
\text { 300-420 Kbp (region A) }\end{array}$ \\
\hline \hline transcription & 3 & 0 \\
\hline translation & 5 & 0 \\
\hline replication & 5 & 1 \\
\hline cell process & 5 & 4 \\
\hline cell structure & 8 & 2 \\
\hline metabolism & 7 & 0 \\
\hline others & 0 & 8 \\
\hline hypothetical protein & 45 & \\
\hline
\end{tabular}

(b) GC3 content of synonymous codons of the orthologous genes is 0.245 or less.

\begin{tabular}{|l|c|c|}
\hline \multicolumn{1}{|c|}{ category of function } & $\begin{array}{c}\text { number of the orthologous } \\
\text { genes }\end{array}$ & $\begin{array}{c}\text { number of the orthologous genes in the } \\
1,320-1,400 \mathrm{Kbp} \text { (region B) }\end{array}$ \\
\hline \hline transcription & 2 & 0 \\
\hline translation & 11 & 6 \\
\hline replication & 0 & 1 \\
\hline cell process & 3 & 0 \\
\hline cell structure & 1 & 1 \\
\hline metabolism & 5 & 0 \\
\hline others & 1 & 5 \\
\hline hypothetical protein & 25 & \\
\hline
\end{tabular}


Chem-Bio Informatics Journal, Vol. 2, No. 2, pp.58-73(2002)

\begin{tabular}{|c|c|c|}
\hline \multicolumn{3}{|c|}{ domain description } \\
\hline$\overline{\mathrm{PH}} 03330$ & hypothetical protein & \\
\hline PH_0331 & hypothetical protein & \\
\hline PH_0332 & Nucleotide Metabolism; Purine metabolism & \\
\hline PH_0334 & hypothetical protein & \\
\hline PH_0335 & hypothetical protein & \\
\hline $\mathrm{PH} 0337$ & hypothetical protein & \\
\hline PH_0340 & pepX related from Caldicellulosiruptor saccharolyticus & \\
\hline PH_0341 & hypothetical protein & \\
\hline PH_0342 & hypothetical protein & \\
\hline PH_-0344 & hypothetical protein & \\
\hline PH_0343 & hypothetical protein & \\
\hline PH_0345 & hypothetical protein & \\
\hline PH_0346 & hypothetical protein & \\
\hline PH_0347 & hypothetical protein & \\
\hline PH_0349 & hypothetical protein & \\
\hline PH_0352 & hypothetical protein & \\
\hline PH_0353 & GTP-binding ERA related & \\
\hline PH_0355 & hypothetical protein & \\
\hline PH_0356 & hypothetical protein & \\
\hline PH_0357 & glunac- 1 -transferase & \\
\hline PH_0359 & hypothetical protein & \\
\hline PH_0360 & hypothetical protein & \\
\hline PH_0362 & hypothetical protein & \\
\hline PH_0363 & hypothetical protein & \\
\hline PH_-0364 & hypothetical protein & \\
\hline PH_0365 & geranylgeranyl hydrogenase related & \\
\hline PH_0366 & hypothetical protein & \\
\hline PH_0367 & hypothetical protein & \\
\hline PH_0368 & glutamine synthetase $(\mathrm{g} \mid \mathrm{n} \mathrm{A})$ & \\
\hline PH_0372 & ribonucleotide reductase (nrd) & \\
\hline PH_0381 & hypothetical protein & \\
\hline PH_0382 & cobalamin (5'-phosphate) synthase (cobS-1) & \\
\hline PH_0383 & hypothetical protein & \\
\hline PH_0384 & hypothetical protein & \\
\hline PH_0385 & cobalamin biosynthesis protein $B$ & \\
\hline PH_-0386 & histidinol-phosphate aminotransferase (hisC) & \\
\hline PH_0388 & UDP-glucose 4-epimerase (galE-2) & \\
\hline $\mathrm{PH}_{-} 0390$ & glucose-1-phosphate thymidylyltransferase (graD-1) & \\
\hline PH_-0391 & hypothetical protein & $300-420 \mathrm{Kbp}$ \\
\hline PH_0393 & hypothetical protein & $($ region $\mathrm{A})$ \\
\hline PH_0394 & hypothetical protein & \\
\hline PH_0397 & hypothetical protein & \\
\hline PH_0400 & polysaccharide biosynthesis protein, putative & \\
\hline PH_0411 & glycosyl transferase & \\
\hline PH_0412 & hypothetical protein & \\
\hline PH_0435 & dTDP-glucose 4,6-dehydratase (rfbB) & \\
\hline PH_0437 & DTDP-4-DEHYDRORHAMNOSE 3,5-EPIMERASE (EC 5.1.3.13) & \\
\hline PH_0438 & DTDP-4-DEHYDRORHAMNOSE REDUCTASE & \\
\hline PH_0442 & polysaccharide biosynthesis protein, putative & \\
\hline PH_0454 & dolichol-P-glucose synthetase & \\
\hline PH_0455 & Lps biosynthesis rfbU related protein & \\
\hline PH_0456 & SUA5 related protein & \\
\hline PH_0458 & hypothetical protein & \\
\hline PH_0459 & adenylosuccinate synthetase (purA) & \\
\hline PH_0460 & COMPETENCE-DAMAGE PROTEIN & \\
\hline PH_0461 & putative, translation initiation factor alF-2BIl translation initiation facto & \\
\hline PH_0463 & DNA MISMATCH RECOGNITION PROTEIN MUTS. & \\
\hline PH_0466 & O-sialoglycoprotein endopeptidase, putative & \\
\hline PH_0467 & hypothetical protein & \\
\hline PH_0468 & hypothetical protein & \\
\hline PH_0469 & hypothetical protein & \\
\hline PH_0470 & hypothetical protein & \\
\hline PH_0471 & DNA repair protein RAD25 & \\
\hline PH_0472 & hypothetical protein & \\
\hline PH_0473 & ATP-dependent protease La (lon) & \\
\hline PH_0477 & hyaluronan synthetase related & \\
\hline PH_0478 & glyceraldehyde-3-phosphate & \\
\hline PH_0479 & hypothetical protein & \\
\hline $\mathrm{PH} 0480$ & 2-haloalkanoic acid dehydrogenase related protein & \\
\hline PH_0481 & CDP-diacylglycerol--glycerol-3-phosphate 3 -phosphatidyltransferase (ogsA) & \\
\hline PH_0482 & hypothetical protein & \\
\hline PH_0483 & hypothetical protein & \\
\hline $\mathrm{PH}_{0} 0484$ & hypothetical protein & \\
\hline PH_0485 & hypothetical protein & \\
\hline PH_0487 & thermostable carboxypeptidase & \\
\hline PH_0488 & hypothetical protein & \\
\hline PH_0491 & hypothetical protein & \\
\hline PH_0493 & stomatin-like protein & \\
\hline PH_0494 & hypothetical protein & \\
\hline PH_0495 & hypothetical protein & \\
\hline PH_0496 & $\mathrm{Na}^{+} / \mathrm{Ca}^{+}$exchanging protein related & \\
\hline $\mathrm{PH}, 0499$ & hypothetical protein & \\
\hline
\end{tabular}

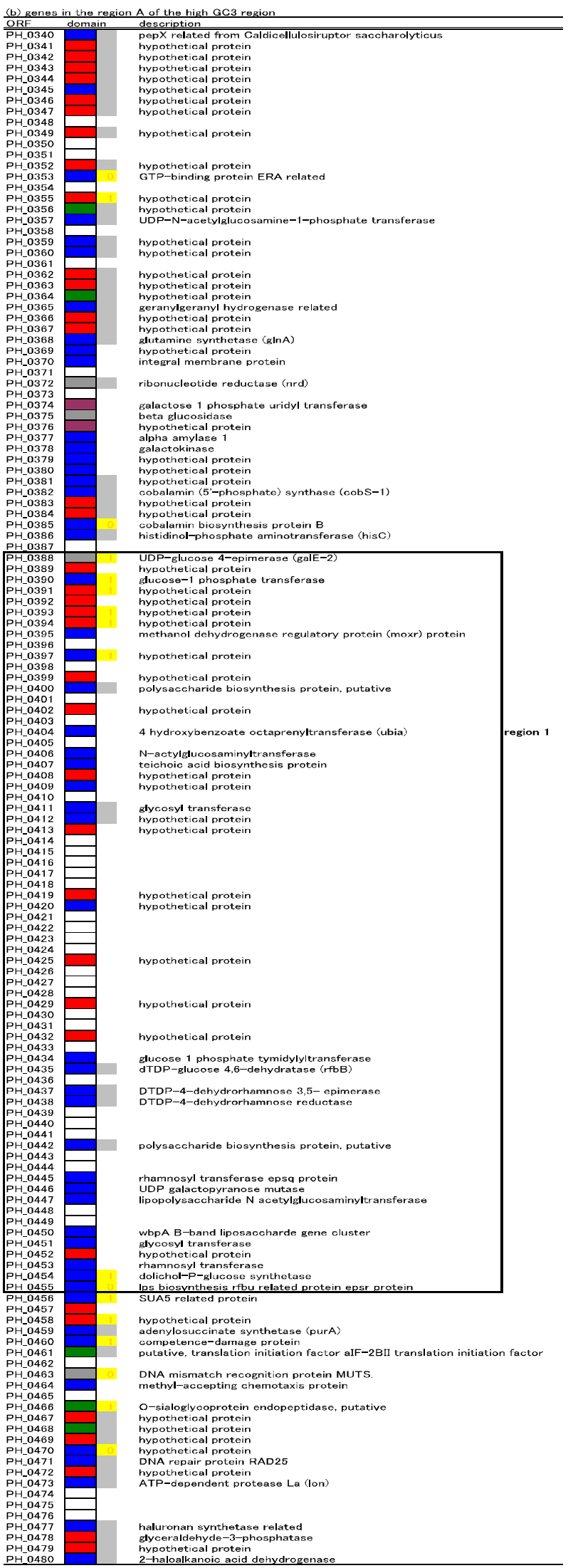

(continued) 

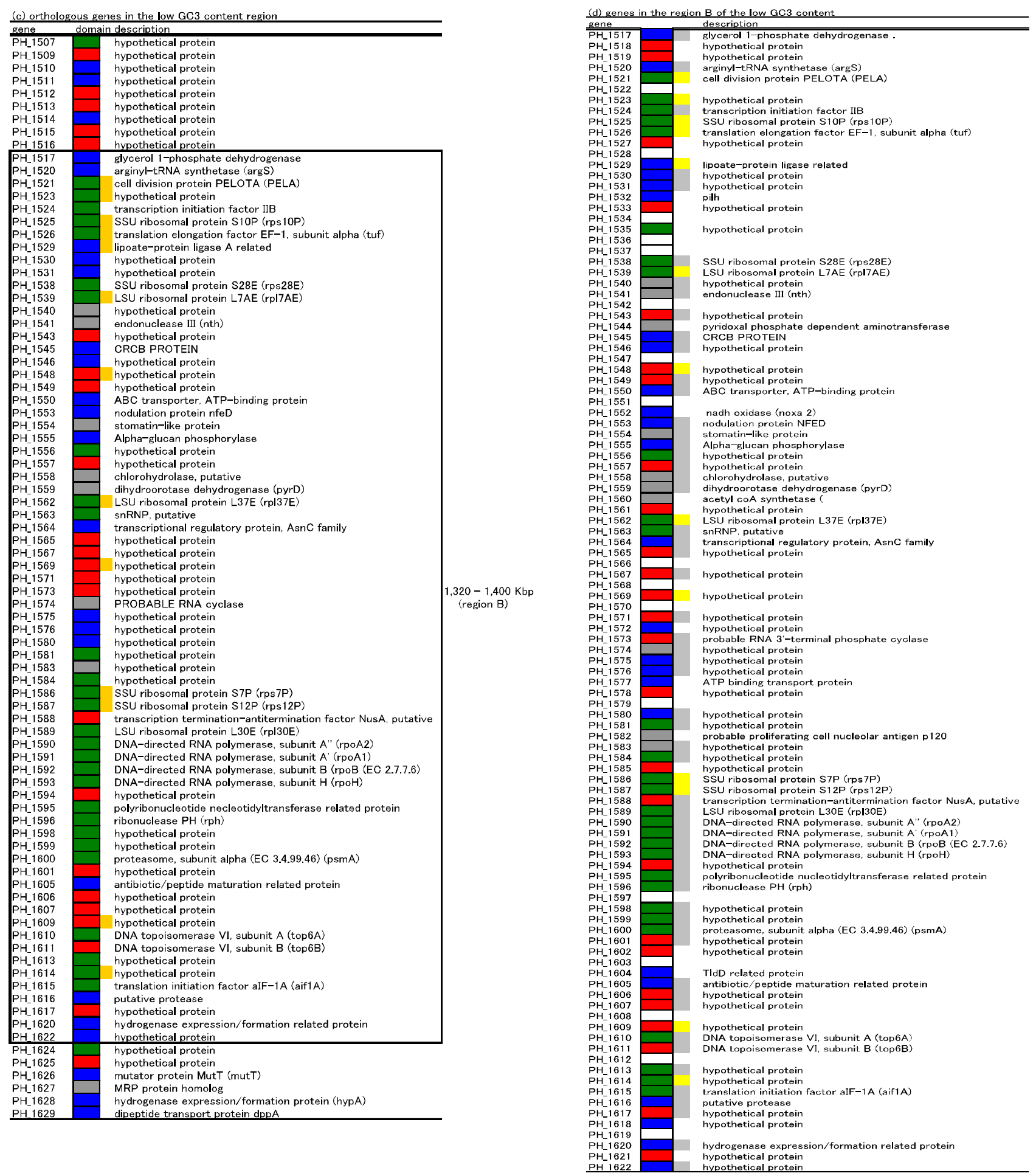

Figure 8. Gene maps of the high and low GC3 content region.

(a) and (b) show the high content region and (c) and (d) show the low content region.

Red blocks indicate the homologues from Archaebacteria. Blue ones indicate those from Eubacteria and Archaebacteria. Green ones indicate those from Eukarya and Archaebacteria. Gray ones indicate those from Eukarya, Eubacteria and Archaebacteria. Violet ones indicate that from Eukarya and Eubacteria. Yellow squares represent the 
genes having the higher GC3 content of synonymous codons, greater than or equal to 0.475 , in region A of the high GC3 content region and those having the lower GC3 content, lower than or equal to 0.245 , in region B of the low GC3 content region. Gray squares represent the orthologous genes. In the region 1 , the orthologous genes were related to cell-wall or lipopolysaccharides and in region $\mathrm{B}$, the orthologous genes were related to translation.

We considered that the region from PH_0388 and PH_0455 in Fig. 8 (b), region 1, would have transferred into $\mathrm{Ph}$ for the following four reasons. In the high GC3 content region, orthologous genes having the higher GC3 content of synonymous codon were located around PH_0388 and PH_0455. In the region A, four genes of function related to cell wall as shown in Table $\overline{2}$ (a), were located at both end of the region 1 . They showed high homology to the genes related to the cell wall or lipopolysaccharides from Streptococcus, which were supposed to have been transferred from $L$.lactis. The genes in this region 1 were homologous to the genes of Eubacteria and Archaebacteria and included 16 genes related to the cell wall or lipopolysaccharides. Moreover, there were many genes without any homologues attributable to the closely related species, Pyrococcus abyssi. Taken together, the region from $\mathrm{PH} \_0388$ to $\mathrm{PH} \_0455$ is suggested to be a lateral region from Eubacteria.

In the low GC3 content region in Fig. $\overline{8}$ (c), region B, six orthologous genes were classified into the category of translation as shown in Table 2 (b). Among these six, five were homologous to genes coding for ribosomal proteins. As shown in Fig. 8 (c), all of the orthologous genes except PH_1529, whose GC3 content of synonymous codons is less than or equal to 0.245 , were homologous to genes from Archaebacteria and Eukarya. It is just conceivable that mutations had been occurring in the nucleotide bases, and as the result of mutation, amino acids were changed or not. In spite of the selection pressure in evolution, changes have been taking effect not on amino acids or proteins but rather on nucleotide bases or the chromosomes. Thus, the smallest deviation from the GC content of the genomic DNA sequence, in comparison with that of other regions, demonstrates that the genes were mostly unaffected in evolution.

Taken together, it is suggested that a region from another species would have transferred into the $300-420 \mathrm{Kbp}$ region of the genomic DNA sequence of Pyrococcus horikoshii OT3, since eight putative lateral genes were located in this region. Additionally, fifteen putative lateral genes were identified in the high GC3 content region.

The index value given to the $\mathrm{G}+\mathrm{C}$ content at the third position of the synonymous codons in the orthologous genes enables us to identify a gene as a lateral gene existing among the genes that would have been affected with respect to on base composition but have not been directly affected with respect to amino acids in evolution. Since such genes could be removed during species relationship analysis, a better phylogenetic tree could be produced that would aid in more properly understanding the phylogenetic relationship. We applied this method to analyze Pyrococcus horikoshii OT3 and identified genes and a region from Eubacteria as the respective lateral genes and a lateral region within the genome DNA sequence.

\section{References}

[1] E. V. Koonin and M. Y. Galperin, Curr. Opin. Genet. Dev., 7, 757-763 (1997).

[2] W. F. Doolittle, Science, 284, 2124-2129 (1999).

[3] R. Muñoz et al., J. Mol. Evol., 46, 432-436 (1998).

[4] M. I. Bellgard and T. Gojobori, Gene, 238, 33-37 (1999).

[5] Y. Kawarabayasi et al., DNA Res., 6, 83-101 (1999). 
[6] H. P. Klenk et al., Nature, 390, 364-370 (1997).

[7] D. R. Smith et al., J. Bacteriol., 179, 7135-7155 (1997).

[8] C. J. Bult et al., Science, 273, 1058-1073 (1996).

[9] Y. Kawarabayasi et al., DNA Res., 5, 55-76 (1998).

[10] http://www.genoscope.cns.fr/Pab/

[11] G. Deckert et al., Nature, 392, 353-358 (1998).

[12] F. Kunst et al., Nature, 390, 249-256 (1997).

[13] C. M. Fraser et al., Nature, 390, 580-586 (1997).

[14] S. Kalman et al., Nat. Gene., 21, 385-389 (1999).

[15] R. S. Stephens et al., Science, 282, 754-759 (1998).

[16] O. White et al., Science, 286, 1571-1577 (1999).

[17] F. R. Blattner et al., Science, 277, 1453-1474 (1997).

[18] R. D. Fleischmann et al., Science, 269, 496-512 (1995).

[19] J. F. Tomb et al., Nature, 388, 539-547 (1997).

[20] R. A. Alm et al., Nature, 397, 176-180 (1999).

[21] C. M. Fraser et al., Science, 270, 397-403 (1995).

[22] R. Himmelreich et al., Nucleic Acids Res., 24, 4420-4449 (1996).

[23] S. G. Andersson et al., Nature, 396, 133-140 (1998).

[24] T. Kaneko et al., DNA Res, 3, 109-136 (1996).

[25] K. E. Nelson et al., Nature, 399, 323-329 (1999).

[26] C. M. Fraser et al., Science, 281, 375-388 (1998).

[27] S. T. Cole et al., Nature, 393, 537-544 (1998).

[28] W. R. Pearson and D. J. Lipman, Proc. Nat. Acad. Sci. USA., 85, 2444-2448 (1988).

[29] J. D. Thompson et al., Nucleic Acids Res., 22, 4673-4680 (1994).

[30] M. Riley, Curr. Opin. Struct. Biol., 8, 388-392 (1998).

[31] A. Muto and S. Osawa, Proc. Natl. Acad. Sci. USA., 84, 166-169 (1987).

[32] G. Bernardi and G. Bernardi, J. Mol. Evol., 22, 363-365 (1985).

[33] C. Médigue, et al., Mol. Microbial., 5, 2629-2640 (1991a)

[34] C. Médigue, et al., J. Mol. Biol., 222, 851-856 (1991b).

[35] F. Bourgoin et al., Gene, 233, 151-161 (1999). 